\title{
Design of Fractional Conversion Ratio Switched Capacitor DC-DC Converters by Using Loop-Connected
}

\author{
Krit Smerpitak $^{\mathrm{a}}$, Sawai Pongswatd ${ }^{\mathrm{a}}$, Teerawat Thepmanee $^{\mathrm{a}}$, Kei Eguchi $^{\mathrm{b}}$ \\ ${ }^{a}$ Faculty of Engineering, King Mongkut's Institute of Technology Ladkrabang \\ Ladkrabang, Bangkok, 10520, Thailand \\ \{klsawai; kskrit \}@kmitl.ac.th \\ ${ }^{\mathrm{b}}$ Department of Information Electronics \\ Fukuoka Institute of Technology \\ 3-30-1 Wajiro-Higashi, Higashi-Ku, Fukuoka 811-0295, Japan \\ eguti@fit.ac.jp
}

\begin{abstract}
A switched-capacitor (SC) DC-DC converter and its loop-connected structure are proposed in this paper. Unlike the conventional series-connected converter, the proposed converter using loop connected provides the output voltage expressed as a combination of multiplication and subtraction. Therefore, the proposed converter can realize various kinds of output voltages as well as circuit size fewer than the conventional converter. Concerning the simple example of the proposed converter, simulation program with SPICE simulations and theoretical analysis are performed to clarify characteristics. The results of SPICE simulations show the following results: (1) The proposed converter can achieve higher power efficiency than the series-parallel type block when the number of capacitors is less than three. (2) In the case of the $2 x$ and $1 / 2 \mathrm{x}$ conversion, the circuit size of the proposed loopconnected converter is less than $80 \%$ of that of the conventional converter. (3) Handy theoretical formulas to estimate power efficiency are obtained by the theoretical analysis. The results of the theoretical analysis are in good agreement with the SPICE simulated results.
\end{abstract}

Keywords: DC-DC converters, switched capacitor circuits, step-up/step-down conversion, bidirectional power flow.

\section{Introduction}

Switched capacitor (SC) DC-DC converter [1]-[6] is one of the most promising converters for mobile equipment, WSN (Wireless Sensor Network) and portable devices, because it can be designed by semiconductor switches and capacitors. The features of the SC DC-DC converter are small volume and light weight. However, in the SC DC-DC converter, the conversion ratio is predetermined by the number of capacitors. The drawback of the SC DC-DC converter is that the conversion ratio cannot be changed linearly. For this reason, several types of circuit topologies have been proposed to realize various conversion ratios. For example, Terada et al and Eguchi et al propose cascade-connected SC DC-DC converter [3],[4], and Chang and Eguchi et al proposed series-connected SC DC-DC converter [1],[2]. By cascading SC DC-DC converters, the cascade-connected converter provides the sum of the output voltage of each converter. However, Terada's converter [3] needs a complicated multiphase timing circuit. On the other hand, by connecting SC DC-DC converters in series, the series- connected converter provides integral multiples of the input or integral divisions of the input. Unlike the cascade-connected converter, Chang's converter [1] can be controlled by two-phase clock pulses. Nevertheless, there is still room for improvement. In this paper, a SC DC-DC converter and its loop-connected structure are proposed. By controlling switches by two-phase clock pulses, the proposed converter supplies the multiplied step-up or step-down voltage to each terminal of output load. Therefore, unlike the conventional converters [1]-[4], the proposed loop-connected converter using bidirectional SC DC-DC converters can provide the output voltage expressed as a combination of multiplication and subtraction. To confirm the validity of the proposed technique, simulation program with SPICE simulations and theoretical analysis are performed. 


\section{Circuit Structure}

\subsection{Conventional Converter}

Fig.1 shows the conventional series-connected converter proposed by Chang [1], Eguchi et al [2], and so on. Since the converter block of Fig.1 (a) is a series-parallel type converter [1],[5] proposed by Ueno et al, the conversion ration $\gamma_{i}(i=1, \ldots, R)$ of the $i$-th converter block is $m$ or $1 / m(m=1, \ldots, N)$. The series-parallel type converter of Fig. 1 (b) consists of $3 N+1$ switches and $N$ capacitors. Table 1 shows the timing of clock pulses for Fig.1 (b). As Table 1shows, the MOS switch is driven by non-overlapped two-phase pulses. By connecting series- parallel type converters, the conventional converter provides the following output voltage:

$$
V_{\text {out }} \cong \prod_{k=1}^{R} \gamma_{k} V_{\text {in }} .
$$

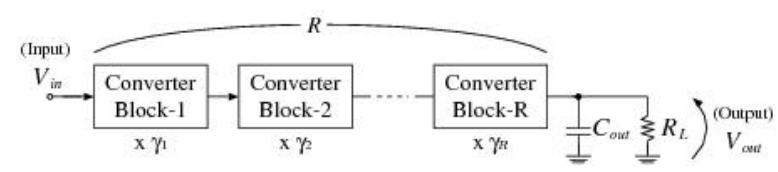

(a)

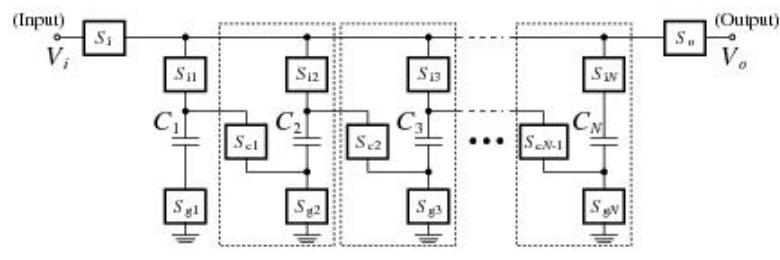

(b)

Fig. 1. Conventional converter using series-parallel converters,

(a) Block diagram, (b) Converter block

Table 1. Timing of clock pulses for the series-parallel converter.

\begin{tabular}{|c|c|c|c|}
\hline $\begin{array}{c}\text { Conversion } \\
\text { Mode }\end{array}$ & State & On & Off \\
\hline \multirow{2}{*}{ Up } & $T_{1}$ & $S_{i}, S_{g 1}, \ldots, S_{g N}$, & $S_{o}, S_{g 1}$, \\
\cline { 2 - 4 } & $T_{2}$ & $S_{i 1}, \ldots, S_{i N}, S_{g 1}$, & $S_{c 1}, \ldots, S_{c N-1}$ \\
\hline \multirow{5}{*}{ Down } & $T_{1}$ & $S_{c 1}, \ldots, S_{c N-1}$ & $S_{i}, S_{g 1}, \ldots, S_{g N}$, \\
& & $S_{i N}, S_{g 1}$, & $S_{i 1}, \ldots, S_{i N}$ \\
& $T_{2}$ & $S_{c 1}, \ldots, S_{c N-1}$ & $S_{o}, S_{g 1}, \ldots, S_{g N}$, \\
& & $S_{o}, S_{g 1}, \ldots, S_{g N}$, & $S_{i 1}, \ldots, S_{i N}$ \\
& & $S_{i 1}, \ldots, S_{i N}$ & $S_{i N}, S_{g 1}$, \\
& & & $S_{c 1}, \ldots, S_{c N-1}$ \\
\hline
\end{tabular}

From Eqs.(1), the series-connected structure of the conventional converter provides step up or down of the input. Although the conventional converter can generate various kinds of output voltages, there is still room for improvement.

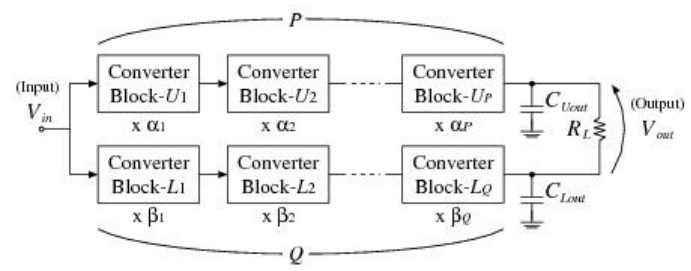

(a)

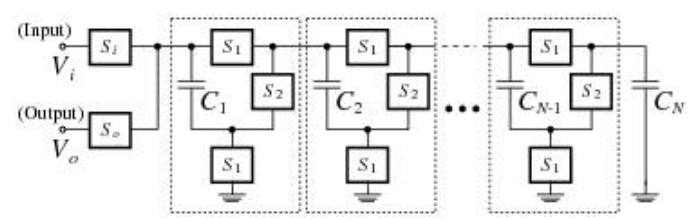

(b)

Fig. 2. Proposed converter using bidirectional DC-DC converters,

(a) Block diagram, (b) Converter block

\subsection{Proposed Converter}

Fig.2 shows the proposed loop-connected converter using bidirectional SC DC-DC converters. The bidirectional DC-DC converter of Fig.2 (b) consists of 3N-1 switches and $\mathrm{N}$ capacitors. Compared with the series-parallel type converter of Fig.1 (b), the structure of the proposed bidirectional DC-DC converter is simple. By connecting bidirectional DC-DC converters, the proposed loop-connected converter provides the following output voltage:

$$
V_{\text {out }} \cong\left(\prod_{i=1}^{P} \alpha_{i}-\prod_{j=1}^{Q} \beta_{j}\right) V_{\text {in }},
$$

where the conversion rations $\alpha_{i}(i=1, \ldots, P)$ and $\beta_{j}(j=1, \ldots$, $Q)$ of the converter block are $m$ or $1 / m(m=1, \ldots, N)$. Unlike the conventional converter, the output voltage of the proposed converter is expressed as a combination of multiplication and subtraction. Fractional conversion ratio of Switched Capacitor DC-DC converters can define by $\alpha_{i}$ and $\beta_{j}$ conversion rations.

\section{Theoretical Analysis}

Concerning power efficiency and output voltage, the theoretical analysis is performed in this section. The theoretical analysis is performed under the conditions: 1) Parasitic elements are negligibly small; and 2) Time constant is much larger than the period of clock pulses. 
Table 2. Timing of clock pulses for the bidirectional converter.

\begin{tabular}{|c|c|c|c|}
\hline $\begin{array}{c}\text { Conversion } \\
\text { Mode }\end{array}$ & State & On & Off \\
\hline \multirow{2}{*}{ Up } & $T_{1}$ & $S_{i}, S_{1}$ & $S_{o}, S_{2}$ \\
\cline { 2 - 4 } & $T_{2}$ & $S_{o}, S_{2}$ & $S_{i}, S_{1}$ \\
\hline \multirow{2}{*}{ Down } & $T_{1}$ & $S_{o}, S_{1}$ & $S_{i}, S_{2}$ \\
\cline { 2 - 4 } & $T_{2}$ & $S_{i}, S_{2}$ & $S_{o}, S_{1}$ \\
\hline
\end{tabular}

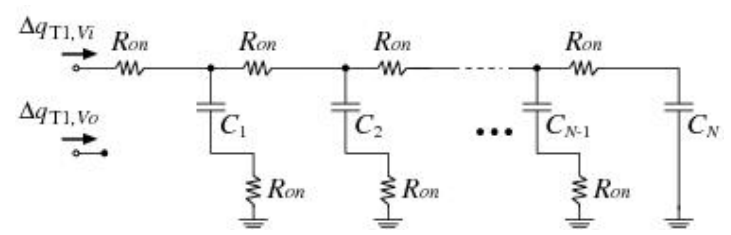

(a)

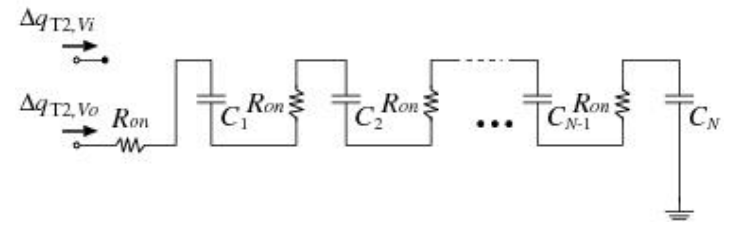

(b)

Fig. 3. Instantaneous equivalent circuits in the case of the step-up conversion, (a) State- $T_{1}$, (b) State- $T_{2}$

\subsection{Converter Block}

First, the characteristic of the proposed converter block is analyzed. Fig. 3 shows the instantaneous equivalent circuits in the case of the step-up conversion, where $R_{o n}$ denotes the on-resistance of the MOS switch. In Fig.3, State $-T_{1}$ is a charging process and State $-T_{2}$ is a transferring process. In Fig.3, the differential value of the electric charge in capacitor $C_{k}(k=1, \ldots, N)$ satisfies

$$
\Delta q_{T_{1}}^{k}+\Delta q_{T_{2}}^{k}=0
$$

where $T=T_{1}+T_{2}, T_{1}=D T$, and $T_{2}=(1-D) T$.

From Eqs.(3), $\Delta q_{T_{i}}^{k}(i=1,2)$ denotes electric charges in the case of State- $T_{i}$ and $D$ is the duty factor. In the case of State- $T_{i}$, the differential values of electric charges in the terminal $V_{i}$ and terminal $V_{o}, \Delta q_{T i, V i}$ and $\Delta q_{T i, V o}$, are obtained by the following equations:

$$
\begin{array}{ll}
\text { State- } T_{1}: & \Delta q_{T_{1}, V_{i}}=\sum_{k=1}^{N} \Delta q_{T_{1}}^{k} \\
\text { and } & \Delta q_{T_{1}, V_{o}}=0
\end{array}
$$

State- $T_{2}$

$$
\Delta q_{T_{2}, V_{i}}=0
$$

and

$$
\Delta q_{T_{2}, V_{o}}=\Delta q_{T_{2}}^{1}=\cdots=\Delta q_{T_{2}}^{N}=0 .
$$

Using (3) - (5), the average input current and the average output current can be expressed as

$$
\begin{aligned}
& \overline{I_{i}}=\frac{\Delta q_{T_{1}, V_{i}}+\Delta q_{T_{2}, V_{i}}}{T}=\frac{\Delta q_{V_{i}}}{T} \\
\text { and } \quad \overline{I_{o}} & =\frac{\Delta q_{T_{1}, V_{o}}+\Delta q_{T_{2}, V_{o}}}{T}=\frac{\Delta q_{V_{o}}}{T},
\end{aligned}
$$

where $\Delta q_{V i}$ and $\Delta q_{V o}$ are electric charges in the terminal $V_{i}$ and the terminal $V_{o}$, respectively. Substituting (3) - (5) for (6), we have the relation between the average input current and the average output current as follows:

$$
\overline{I_{i}}=-N \overline{I_{o}},
$$

where

$$
\Delta q_{V_{i}}=-N \Delta q_{T_{1}}^{1} \quad \text { and } \quad \Delta q_{V_{o}}=-\Delta q_{T_{1}}^{1} .
$$

The consumed energy in one period. In Fig.3, the consumed energy in one period can be expressed as

$$
W_{T}=W_{T_{1}}+W_{T_{2}},
$$

where

$$
\begin{aligned}
W_{T_{1}} & =\sum_{k=1}^{N-1} \frac{R_{o n}}{T_{1}}\left(\Delta q_{T_{1}}^{k}\right)^{2}+\sum_{l=1}^{N}\left\{\frac{R_{o n}}{T_{1}}\left(\sum_{k=l}^{N} \Delta q_{T_{1}}^{k}\right)^{2}\right\} \\
& =\frac{(N-1) R_{o n}}{D T}\left(\Delta q_{T_{1}}^{1}\right)^{2}+\sum_{k=1}^{N} \frac{k^{2} R_{o n}}{D T}\left(\Delta q_{T_{1}}^{1}\right)^{2}
\end{aligned}
$$

and

$$
W_{T_{2}}=\frac{N R_{o n}}{T_{2}}\left(\Delta q_{T_{2}}^{1}\right)^{2}=\frac{N R_{o n}}{(1-D) T}\left(\Delta q_{T_{1}}^{1}\right)^{2}
$$

Therefore, we have the consumed energy as follows:

$$
W_{T}=\frac{(N-1) R_{o n}}{D T}\left(\Delta q_{V_{o}}\right)^{2}+\sum_{k=1}^{N} \frac{k^{2} R_{o n}}{D T}\left(\Delta q_{V_{o}}\right)^{2}+\frac{N R_{o n}}{(1-D) T}\left(\Delta q_{V_{o}}\right)^{2} .
$$

The consumed energy $W_{T}$ is defined as

$$
W_{T}:=\left(\frac{\Delta q_{V_{o}}}{T}\right)^{2} \cdot R_{S C} \cdot T,
$$

where $R_{S C}$ is called the SC resistance. Therefore, from (9) and (10), the SC resistance in the case of the step-up conversion can be obtained as

$$
R_{S C}=\frac{R_{o n}(N-1)(1-D)+R_{o n} N D+R_{o n}(1-D) \sum_{k=1}^{N} k^{2}}{D(1-D)} .
$$

By combining (7) and (11), we have the equivalent circuit 
in the case of the step-up conversion as follows:

$$
\left[\begin{array}{c}
\bar{V}_{i} \\
\bar{I}_{i}
\end{array}\right]=\left[\begin{array}{cc}
\frac{1}{N} & 0 \\
0 & N
\end{array}\right]\left[\begin{array}{cc}
1 & R_{S C} \\
0 & 1
\end{array}\right]\left[\begin{array}{c}
\overline{V_{o}} \\
-\overline{I_{o}}
\end{array}\right]
$$

because the equivalent circuit of the SC power converters can be expressed by the determinant using the Kettenmatrix.

On the other hand, the equivalent circuit in the case of the step-down conversion can be obtained by the same method. In the case of the step-down conversion, the equivalent circuit can be expressed by the following determinant:

$$
\left[\begin{array}{c}
\overline{V_{i}} \\
\overline{I_{i}}
\end{array}\right]=\left[\begin{array}{cc}
N & 0 \\
0 & \frac{1}{N}
\end{array}\right]\left[\begin{array}{cc}
1 & R_{S C} \\
0 & 1
\end{array}\right]\left[\begin{array}{c}
\overline{V_{o}} \\
-\overline{I_{o}}
\end{array}\right]
$$

where

$$
R_{S C}=\frac{R_{o n} N(1-D)+R_{o n}(N-1) D+R_{o n} D \sum_{k=1}^{N} k^{2}}{N^{2} D(1-D)} .
$$

\subsection{Loop-Connected Structure}

From (11) - (14), the equivalent circuit of the proposed loop-connected converter can be expressed by Fig.4. In Fig. $4, \alpha_{i}$ is the conversion ratio of the $i$-th $(i=1, \ldots, P)$ upper converter block, $\beta_{j}$ is the conversion ratio of the $j$-th $(j=1$, $\ldots, Q)$ lower converter block, $R_{s c U i}$ is the SC resistance of the $i$-th upper converter block, and $R_{s c L j}$ is the SC resistance of the $j$-th lower converter block. From Fig.4, we have the power efficiency and the output voltage as follows:

$$
\eta=\frac{R_{L}}{R_{L}+\sum_{i=1}^{P}\left\{\prod_{j=i+1}^{P}\left(\alpha_{j}\right)^{2}\right\} R_{s c U_{i}}+\sum_{i=1}^{Q}\left\{\prod_{j=i+1}^{Q}\left(\beta_{j}\right)^{2}\right\} R_{s c L_{i}}}
$$

and

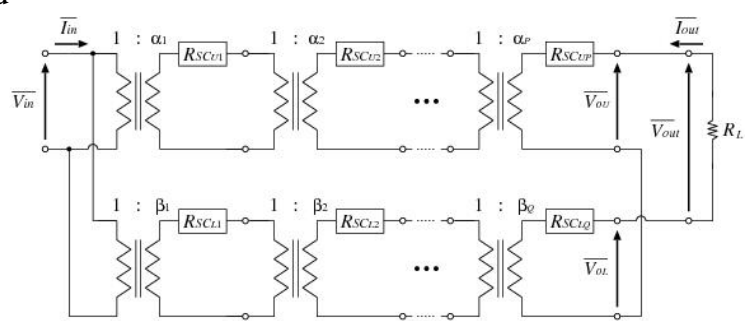

Fig. 4. Equivalent circuit of the proposed converter

$$
V_{\text {out }}=\eta\left\{\left(\prod_{k=1}^{P} \alpha_{k}\right)-\left(\prod_{k=1}^{Q} \beta_{k}\right)\right\} V_{\text {in }},
$$

where

$$
\begin{aligned}
& \prod_{j=i+1}^{P}\left(\alpha_{j}\right)^{2}=1 \quad(\text { if } \quad i+1>P) \\
& \text { and } \\
& \prod_{j=i+1}^{Q}\left(\beta_{j}\right)^{2}=1 \quad(\text { if } \quad i+1>Q)
\end{aligned}
$$

\section{Simulation}

To confirm the validity of the theoretical analyses, SPICE simulations were performed with $2 \mathrm{x}$ converter and 1/2x loop connected converter. The circuit structure can be shown by Fig. 5 that defines parameters under condition $V_{\text {in }}=2 \mathrm{~V}, \quad c_{1}=c_{2}=0.22 \mu \mathrm{F}, R_{L}=10 \mathrm{k} \Omega$. In addition, Fig. 6 and 7 show the simulation result and power efficiency.

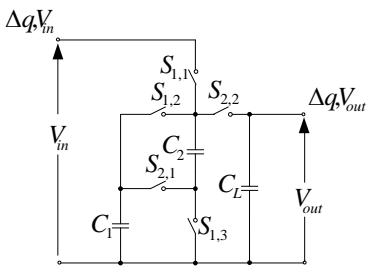

(a)

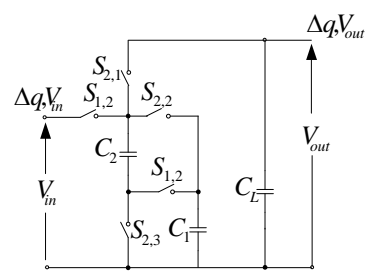

(b)
Fig. 5. Circuit structure of proposed SC DC-DC converter (a) 2x step up converter (b) 1/2x step down converter

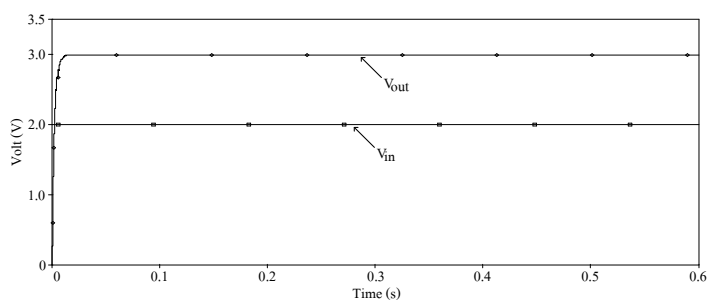

Fig. 6. Simulation result of $2 \mathrm{x}$ and $1 / 2 \mathrm{x}$ loop connected where $V_{\text {in }}=2 \mathrm{~V}$

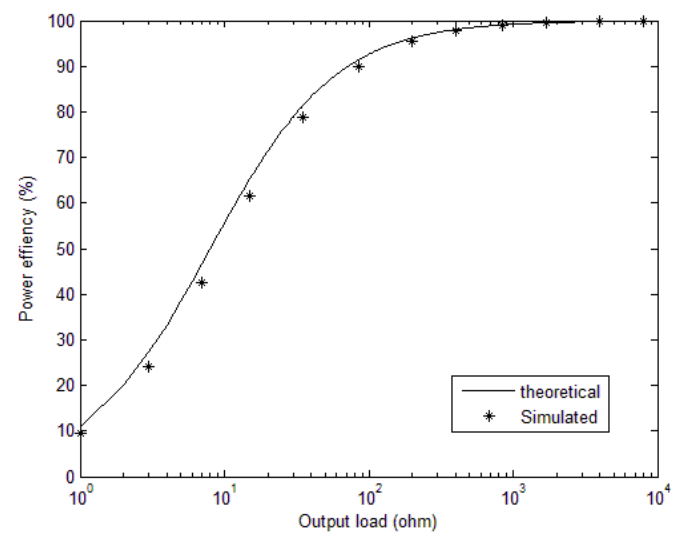

Fig. 7. Power efficiency as function of output load $R_{L}$

Fig.6 shows the simulated output voltage from $2 \mathrm{x}$ and $1 / 2 \mathrm{x}$ loop connected converter that input $\mathrm{Vin}$ is $2 \mathrm{~V}$. 
Simulated results correspond well with theoretical results, For this reason, derived theoretical formulas will be helpful to design the SC converter that can define multi fraction conversion ratio. In additional, the power efficiency is shown by Fig.7 that shows of $2 x$ and 1/2x loop connected converter as a function of output load $R_{L}$. The power efficiency is a ratio of output load and SC resistance which follow by Eqs.(15). In other words, the proposed converter can realize the input range that is wider than conversional converter by connect converter as loop connected. Table 3 shows the number of circuit elements to achieve the $3 / 2 x$ conversions. As Table 3 shows, the proposed loop-connected converter can realize complex conversion ratios in the small number of circuit elements.

Table 3. Number of circuit elements

\begin{tabular}{|c|l|c|c|}
\hline $\begin{array}{c}\text { Conversion } \\
\text { Ratio }\end{array}$ & \multicolumn{1}{|c|}{ Converter Type } & Switch & Capacitor \\
\hline $3 / 2 \times$ & Proposed converter & 10 & 4 \\
\cline { 2 - 4 } 3 & Conventional converter & 16 & 5 \\
\hline
\end{tabular}

\section{Conclusions}

In this paper, a loop-connected converter using bidirectional switched capacitor DC-DC converters has been proposed. The validity of the circuit design was confirmed by SPICE simulations, theoretical analysis, and experiment. The results of the SPICE simulations and theoretical analysis showed the following results: (1) The proposed loop-connected converter can achieve higher power efficiency than the conventional series- parallel-type converter when the number of capacitors is less than three. (2) The theoretical equations will be helpful to design the proposed converter, because theoretical results were in good agreement with the SPICE simulated results. The proposed technique will apply as driver circuit for battery charger of WSN(wireless Sensor Network) and electrical power transfer between various power sources.

\section{References}

(1) Y.H. Chang : "Variable-Conversion-Ratio Switched- CapacitorVoltage- Multiplier/ Divider DC-DC Converter," IEEE Trans. Circuits and Systems I, vol.58, Issue 8, pp. 1944-1957, Aug. 2011.

(2) K. Eguchi, F. Ueno, H. Zhu, and T. Tabata : "Design of a cell-network type SC DC-DC converter for mobile equipments," in Proc. IEEE Region 10 Conference, TENCON 2004, 2004, pp. 37-40.

(3) S. Terada, I. Oota, K. Eguchi, and F. Ueno : "Switched-capacitor (SC) DC-DC converter with fine tune control outputs," in Proc. the 6th International Caribbean Conference on Devices, Circuits and Systems, 2006, pp. 233-236.
(4) K. Eguchi, S. Pongswatd, K. Tirasesth, H. Sasaki, I. Oota, T. Inoue : "A switched-capacitor-based serial DC-DC converter using clean energy power supplies," International Journal of Innovative Computing Information and Control, vol. 7, no. 6, pp. 3485-3498, June 2011

(5) M.D. Seeman, and S.R. Sanders : "Analysis and Optimization of Switched-Capacitor DC-DC Converters," IEEE Trans. Power Electronics, vol.23, no.2, pp. 841-851, Aug. 2008.

(6) Sawai Pongswatd, Krit Smerpituk, Prasit Julsereewong, Kei Eguchi, Hirofumi Sasaki : "Design of 2x and 1/2x Fraction Conversion Ratio for SC DC-DC Converters", The 2013 ECTI International Conference on Electrical Engineering/Electronics, Computer, Telecommunications and Information Technology (ECTI-CON 2013), May 15-17, 2013, Krabi, Thailand. 